\title{
Polycystic Ovarian Syndrome Trend in a Nutshell
}

\author{
Stephanie B Mohammed ${ }^{1 *}$, B Shivananda Nayak ${ }^{1}$
}

\begin{abstract}
Objectives: To identify the trend occurring in the diagnosis, prognosis, and therapy treatment of women diagnosed with polycystic ovarian syndrome (PCOS) and to highlight the importance of the accumulated and accepted decisions made.

Methods: To perform a quality assessment on the literature available from specific search engines throughout the years 1800-2017. Results: PCOS affects women physically, psychologically, metabolically, endocrinologically and reproductively. Women are identified from the basic cardinal features classifiable with the condition from which they are then recommended to have diagnostic testing. To date, these involve the use of an ultrasound for the identification of immature follicles and biochemical testing of ovarian hormones and corresponding blood test in relation to the signs and symptoms exhibited. PCOS has shown to be present from the pre-adolescent stage throughout adolescent into adulthood and it is observed to affect women regardless of their ethnicities and demographics.

Conclusion: Presently there are various proposed treatments available for the management of PCOS which includes lifestyle modification, administration of pharmaceutical agents and surgical procedures.

Keywords: PCOS, Symptoms, Diagnosis, Treatments
\end{abstract}

\section{Introduction}

What Is Polycystic Ovarian Syndrome?

Polycystic ovarian syndrome (PCOS) is considered to be a trending topic in research worldwide for countless of intriguing scientists, researchers, medical professionals and most of all the women diagnosed with the condition.

Several definitions surround the terminology of PCOS as it is considered heterogeneous in nature with an overall negative impact on the health status of women. While some women may present symptomatic and others asymptomatic, the condition is believed to affect women physically, psychologically, metabolically, endocrinologically and reproductively.

As with any trending topic, countless of research have been conducted and consensus have been held to summarize the major findings from which would be considered acceptable for the proper diagnosing process.

\section{Methods}

A critical analysis of the literature on PCOS was conducted. The search engines PubMed and Google Scholar were searched and summarized throughout the years 18002017 based on the following word text PCOS, treatment, ethnicities, consensus, statistics and aetiology. These texts were added together or independent of each other. The objective of this review was to identify and summarize the trends and patterns established on PCOS in a nutshell. Papers chosen must have followed the code of ethics and patient consent had to be received. Only papers written in English were considered for this review.

\section{Results}

One of the most outstanding proposed consensus was published in 2002, in which a concise scheme for the diagnosis of PCOS was presented. In this proposition, Homburg suggested a process for the examination of PCOS by which women should first be assessed for the symptoms of menstrual irregularities, hirsutism, acne and infertility complications. Once there was an existence or potential of disturbances in any of these, he then suggested it be verified with the use of an ultrasound. If a positive result was obtained then the tests for biochemical parameters of elevated testosterone, luteinizing hormone (LH), free androgen levels and fasting glucose should be performed to assist in the regimen (1).

Since then, numerous of highly recognized and cited consensus have been established in providing guidelines for the signs and symptoms experienced by this dreadful condition.

In 2004 a revised Rotterdam consensus that was previously held in 2003 endorsed by the European Society for Human Reproduction and Embryology (ESHRE) and the American Society for Reproductive Medicine (ASRM) took action in establishing a diagnostic criterion as well. The outcome highlighted the diverse signs and symptoms associated with PCOS which included ovarian dysfunction, basic cardinal features of hyperandrogenism, polycystic ovaries morphology and clinical manifestations of menstrual irregularities, signs of androgen excess, obesity, insulin resistance (IR) and elevated LH present in women with PCOS (2). Then, in 2009, similar results were 
presented again from a systematic review published by the Androgen Excess and PCOS (AE-PCOS) Society (3).

The importance of establishing fundamental signs and symptoms from these consensuses became the foundation from which women with PCOS would be identified. One consensus highlighted the importance of inconsistency in these signs. As in 2012 the National Institute of Health Evidence-based Methodology Workshop on PCOS expressed that women may present with or without androgen excess, ovulatory dysfunction and polycystic ovarian morphology (4).

Another major concern not addressed was the inconsistency of symptoms expressed by women of differing age categories as it was not confirmed whether women of a younger age exhibited similar signs when compared to the older women.

To address this gap, in 2012 another consensus took action in highlighting PCOS among different groups of women. The outcome exposed that adolescents exhibited dissimilar results when compared to older reproductive women as, some amenorrhea and oligomenorrhea women were considered to ovulate. It also highlighted that some women with PCOS may not have exhibited the features identifiable with the previous consensus as stated (5).

PCOS was therefore observed to be present in women regardless of exhibiting all the physical findings as the use of clinical measurements were able to provide true confirmations. However, they still had to be either or, but not confined to all the symptoms as expressed.

To date the basic features, consist of abdominal pain, acne, menstrual irregularities and hirsutism on one or more body parts such as the upper lip, chin, breasts, chest between breast, back, belly, upper arms and upper thighs (6). Some women are also observed to have high body mass index (BMI) thus leading to obesity (7).

PCOS would also stimulate depression and reduce the quality of life as women may indulge in unhealthy diets, have cravings for high GI diets and fall short in exercise (8-11).

Their metabolic and endocrine results can lead to changes in LH and FSH levels, elevated testosterone and oestrogen values and decrease sex-binding globulin (SHBG) (12). Other health complications associated with PCOS are IR, T2D, hypertension (13), coronary artery disease, cerebral vascular disease (14) and overall the metabolic syndrome.

The use of a trans-abdominal or trans-vaginal ultrasound proved to be useful for observing the presence of the ovary size (i.e. the volume) and the number of preantral follicles (15).

The morphological hallmark of PCOS remains to be bilaterally enlarged ovaries with multiple cysts (16). Yet in viewing ovaries, the screening for capsular thickening, subcapsular follicular cysts and hyperplasia of the theca interna are also advised to be viewed (17). The ovary report should also highlight ovarian volume and antral follicle counts (18). Typical PCOS appearance $>10$ cysts nearing $9 \mathrm{~mm}$ in diameter to 12 follicles of 2-9 $\mathrm{mm}$ with increase ovarian size $>10 \mathrm{~cm}^{3}$ (19).

\section{Statistics on Polycystic Ovarian Syndrome and Aetiology of Polycystic Ovarian Syndrome}

The occurrences of PCOS have shown to affect 5\%-10\% of women of reproductive age (20). Statistics also identified approximately 5 million women in the Unites States living with PCOS with a cost on average of 4 billion US dollars annually for just the management of PCOS (4).

The experience has shown to have a genetic trend as it was found present in first relatives since the ratio of PCOS in mothers and sisters of patients with PCOS were observed to be $24 \%$ and $32 \%$ respectively (21).

There have also been evidence of PCOS in 13\%-25\% of women with epilepsy (22).

The aetiology of PCOS to date remains blind however it is believed that puberty has been the onset of the syndrome in girls at a perimanachael age. Girls between the ages of 11-18years showed the presence of hyperandrogenism, high gonadotropin levels and metabolic variations (23). Along with PCOS found in adolescents, research also showed it presented it in preadolescent ages 9-12 years (24). PCOS then follows through and remains common in women 18-25 years (25). With an unknown aetiology, the treatment methods recommended are all based on the varying symptoms of each women.

\section{Ethnicities of PCOS Occurrence}

PCOS affects women of all ethnicities. It has been observed in Caucasian, Caribbean-Hispanic, African, American, Native American (26), European, Maori, Pacific Island, Indian, Sri Lanka, African Negro, Iranian, Iraqi (27), NonHispanic White (28), Middles East, Asians (29), Chinese (30) and Japanese (31) to name a few.

Some of the most outstanding features showed that Caucasian women had more adverse cardiovascular disease (CVD) profile when compared with the Middle East (29), Sri Lankans exhibited high fasting plasma homocysteine levels which correlated with fasting insulin, placing women at risk for atherosclerosis (32), Caribbean Hispanic had high IR compared to Non-Hispanic White (28), Maori and Pacific Island with increase obesity and risk for CVD and PCOS most seen in Indian women (27), Chinese at risk for impaired glucose tolerance and diabetes mellitus (30).

The ethnic background and environmental factors all contribute to the major risk factors associated with having PCOS. One of the major risk factors has shown to be the metabolic syndromes which are abnormalities in waist circumference, HDL-C, blood pressure and glucose (33) which can lead to obesity and T2DM. According to a systematic review of CVD risk factors, women with obesity, cigarette smoking, dyslipidaemia (34), hypertension, impaired glucose tolerance and 
subclinical vascular diseases are at a risk for CVD (35). Recent work showed that women with PCOS were seen at increased risk for T2D, gestational diabetes, pregnancy related complications, venous thromboembolism, cerebrovascular and cardiovascular events (36).

Women with PCOS are not only at the risk for complications but a reduction in the quality of their life overall is decreased. They experience physiological disturbances, depression, anxiety, lower degree of sex life, and a lot of stress $(37,38)$.

\section{What Are the Therapy Treatments Recommended for Polycystic Ovarian Syndrome?}

The aetiology of PCOS remains uncertain and so treatments are based on the presenting symptoms. One of the major concerns of PCOS is of infertility problem with women having PCOS. PCOS is viewed to be one of the leading causes of infertility in young child-bearing women. Anovulation and pregnancy complications such as early loss and multiple miscarriages are also some of the difficulties associated with this dreadful condition.

Some of the pharmaceutical agents recommended include various hormonal agents such as anti-androgens such as cyproterone found in Diane 35, Androcur and cyproterone acetate; clomiphene citrate for ovulation found in Clomid and Serophene; birth control which contains ethinyl estradiol for preventing ovulation such as Yasmin, Diane 35, Yaz; anticoagulant such as Xarelto; Corticosteroid such as Prednisolone or relieve inhaler such as Ventolin; tonics for female menarche such as Femiton; treatment for endometriosis such as Zoladex and medications for controlling blood sugar such as Glucophage and metformin.

Contraceptives have been highly recommended to women with PCOS where pregnancy was not desired which proved to be effective in treating hyperandrogenism and it provided endometrial protection since it contains estrogen and progestin (39).

Treatment of PCOS with clomiphene citrate has shown to be promising for patients wishing to improve the rate of live births. Together metformin and clomiphene indicates to improve insulin sensitivity yet in a study conducted in 2007, the study recommended the use of clomiphene alone as first-line therapy in infertility of PCOS as extendedrelease metformin alone was not considered significant (40). Contrary to this, other research has reported benefits of metformin especially in combination with clomiphene (41). Meta-analysis study observed metformin as an effective treatment for anovulation and higher ovulation rates when combined with clomiphene (42).

Research showed dating from the year 1979-1991, 250 women who were diagnosed with PCOS were treated with electrocautery. This method proved successful ovulation in $92 \%$ and $84 \%$ increases in pregnancy in women whose main cause was infertility. Additionally, when the nonresponders were administered clomiphene citrate (CC)

\section{Review Highlights}

- PCOS affects the psychologically, physically, metabolically, endocrinologically and reproductively states in women. Some women may present symptomatic while others asymptomatic. The most recommended identification for PCOS is with the use of both an ultrasound and biochemical confirmations.

- Polycystic ovarian syndrome has shown to be present from preadolescent to adulthood.

- Polycystic ovary syndrome has shown to be present in Caucasian, Caribbean-Hispanic, African, American, Native American, European, Maori, Pacific Island, Indian, Sri Lanka, African Negro, Iranian, Iraqi, Non-Hispanic White, Middles East, Asians, Chinese and Japanese.

- The various proposed treatments for PCOS to date include lifestyle modification such as diet and exercise, administration of pharmaceutical agents such as clomiphene citrate, gonadotropins and insulin-sensitizing agents and surgical procedures such as laparoscopy and assisted reproduction techniques.

there was an $89 \%$ increase. However, the rate of success was determined by the body weight of each woman as the results were dissimilar for slim when compared with obese (43).

Later on when a consensus was conducted, CC was recommended as the first line of treatment for induction of ovulation. The second line of treatment was then exogenous gonadotropins and insulin sensitizers such as metformin, biguanide and thiazolidinedione. The other recommended treatments were surgical procedures such as laparoscopic ovarian surgery (LOS) and assisted reproduction techniques (ART) such as invitro fertilization and homologous artificial insemination $(44,45)$.

Additional to this, intervention studies have shown how controlled diets improved ovulation (46) and when exercise was added to these diets, it improved menstrual cycles with decreasing testosterone levels (47).

Weight loss have played an important role as it was reported to improve ovulation rates (47) and even a 5\% loss of body weight proved clinically significant.

\section{Conclusion}

Overall as seen in Figure 1 women who present with PCOS are evaluated based on their signs and symptoms, from which they are recommended to undergo an ultrasound and biochemical testing. They are then advised on their lifestyle alterations and recommended pharmacological or surgical treatment if required.

\section{Ethical Issues}

Not applicable.

\section{Conflict of Interests}

The authors declare no conflicts of interests.

\section{Financial Support}

None. 


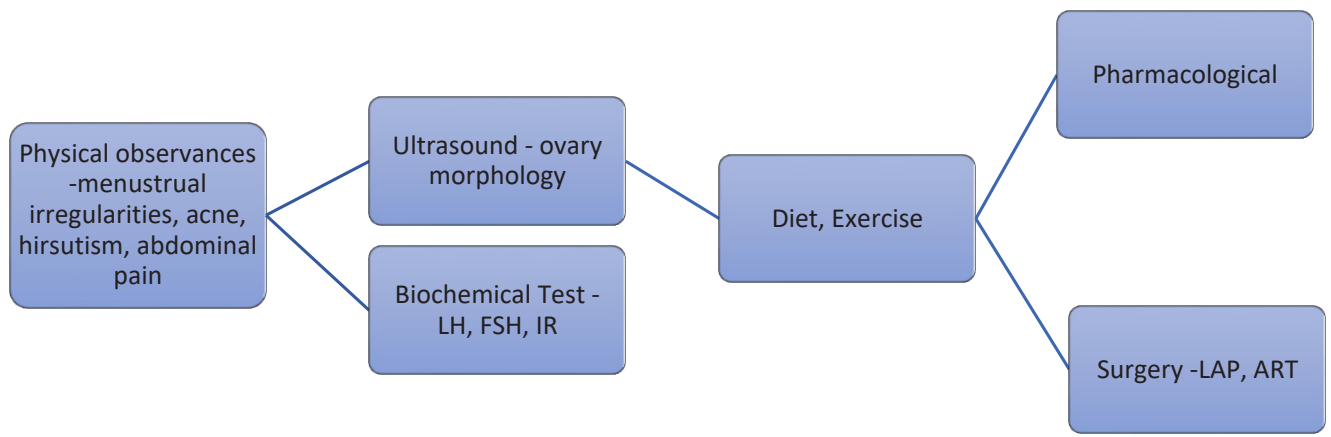

Figure 1. Showing the Pathway From Identification of PCOS to Treatment.

\section{References}

1. Homburg R. What is PCOS? A proposal for a consensus on the definition and diagnosis of polycystic ovarian syndrome. Hum Reprod. 2002;17(10):2495-2499

2. The Rotterdam ESHRE/ASRM-Sponsored PCOS Consensus Workshop Group. Revised 2003 consensus on diagnostic criteria and long term health risks related to polycystic ovary syndrome. Hum Reprod. 2004;19:41-47. doi:10.1016/j.fertnstert.2003.10.004.

3. Azziz R, Carmina E, Dewailly D, et al. The Androgen Excess and PCOS society criteria for the polycystic ovary syndrome: the complete task force report. Fertil Steril. 2009;91(2):456-488. doi: 10.1016/j.fertnstert.2008.06.035.

4. Johnson T, Kaplan Z, Ouyang P, et al. National Institute of Health Evidence-based Methodology Workshop on Polycystic Ovarian Syndrome. Bethesda: National Institute of Health; 2012:1-4.

5. Fauser BCJ, Tarlatzis BC, Rabar RW, et al. Consensus on women's health aspects of polycystic ovary syndrome (PCOS): The Amsterdam ESHRE/ASRM-Sponsored $3^{\text {rd }}$ PCOS Consensus Workshop Group. Fertil Steril. 2012;97(1):28-38e25. doi: 10.1016/j.fertnstert.2011.09.024

6. Pedersen SD, Brar S, Faris P, et al. Polycystic ovary syndrome. Validate questionnaire for use in diagnosis. Canadian Family Physician. 2007;53(6):1041-1047.

7. Azziz R, Woods KS, Reyna R, et al. The prevalence and features of the polycystic ovary syndrome in and unselected population. J Clin Endocrinol Metab. 2004;89(6):27452749. doi:10.1210/jc.2003-032046

8. Mohammed S, Nayak S. Exploration of ovarian hormones, diet and lifestyle of women with polycystic ovarian syndrome. Int J Curr Adv Res. 2017;6(1):1671-1675.

9. Sedighi S, Akbari SAA, Afrakhteh M, et al. Comparison of lifestyle in women with polycystic ovary syndrome and healthy women. Glob J Health Sci. 2015;7(1):228-234. doi: 10.5539/gjhs.v7n1p228

10. Altieri P, Cavazza C, Pasqui F. Dietary habits and their relationship with hormones and metabolism in overweight and obese women with polycystic ovarian syndrome. Clin Endocrinol. 2013;78(1):52-59. doi:10.1111/j.13652265.2012.04355.x

11. Misir A, Bnjari I, Loncar I. Comparison of diet in women of reproduce age with and without diagnosed polycystic ovarian syndrome-pilot study. Medicinski Pregled. 2016;69(9-10):274-280.

12. Udoff LC, Adashi EY. Polycystic ovarian disease: current insights into an old problem. Journal of Adolescent Gynaecology. 1996;9:3-8. doi: 10.2298/MPNS1610274M.
13. Apridonidze T, Essah PA, Iuorno MJ, et al. Prevalence and characteristics of the metabolic syndrome in women with polycystic ovary syndrome. J Clin Endocrinol Metab. 2005;90(4):1929-1935. doi: 10.1210/jc.2004-1045

14. Wild S, Pierpoint T, McKeigue P, et al. Cardiovascular disease in women with polycystic ovary syndrome at long-term follow-up: a retrospective cohort study. Clin Endocrinol. 2000;52(5):595-600.

15. Balen AH, Laven JSE, Tan SL, et al. Ultrasound assessment of the polycystic ovary. International consensus definitions. Hum Reprod Update. 2003;9(6):505-514. doi:10.1093/ humupd/dmg044.

16. Faure N, Prat X, Bastide A, et al. Assessment of ovaries by Magnetic resonance imaging in patients presenting with polycystic ovarian syndrome. Hum Reprod. 1989;4(4):468467. doi:10.1093/oxfordjournals.humrep.a136.

17. Biggs JS. Polycystic ovarian disease-current concepts. Australian and New Zeeland Journal of Obstetrics and Gynaecology. 1981;21(1):26-36. doi:10.1111/j.1479828X.1981.tb00120.x

18. Lee TT, Rausch ME. Polycystic ovarian syndrome: role of imaging in diagnosis. Radio Graphics. 2012;32(6):16431657. doi:10.1148/rg.326125503.

19. Lass A, Brinsden PR. The role of ovarian volume in reproductive medicine. Hum Reprod. 1999;5(3):256-266. doi:10.1093/humupd/5.3.256.

20. Knochenhauer ES, Key TJ, Khasar-Miller M, et al. Prevalence of the polycystic ovary syndrome in unselected black and white women of the south-eastern United States: a prospective study. J Clin Endocrinol Metab. 1998;83:30783082. doi: 10.1210/jcem.83.9.5090

21. Kahsar-Miller MD, Nixon C, Boots LR, et al. Prevalence of PCOS om first-degree relatives of patients with PCOS. Fertil Steril. 2001;75:53-58. doi:10.1016/S0015-0282(00)01662-9.

22. Herzog AG, Schachter SC. Valporate and the polycystic ovarian syndrome: final thoughts. Epilepsia. 2001;42(3):311315. doi:10.1046/j.1528-1157.2001.33500.x.

23. Apter D. Endocrine and metabolic abnormalities in adolescents with a PCOS-like condition: consequence for adult reproduction. Trends Endocrinol Metab. 1998;9(2):58-61. doi:10.1016/S1043-2760(98)00020-4.

24. Bronstein J, Tawdekar S, Liu Y, et al. Age of onset of polycystic ovarian syndrome in girls may be earlier than previously thought. J Pediatr Adolesc Gynecol. 2011;24(1):15-20. doi: 10.1016/j.jpag.2010.06.003.

25. Michelmore KF, Balen AH, Dunger DB, et al. Polycystic ovaries and associated clinical and biochemical features in young women. Clin Endocrinol. 1999;51(6):779-786. doi: 
10.1046/j.1365-2265.1999.00886.x.

26. Cronin L, Guyatt G, Griffith L, et al. Development of Healthrelated quality of life. Questionnaire (PCOSQ) for women with Polycystic ovarian syndrome. J Clin Endocrinol Metab. 1998;83(6):1976-1987. doi: 10.1210/jcem.83.6.4990

27. Williamson K, Gunn AJ, Johnson N, et al. The impact of ethnicity on the presentation of PCOS. Australian and New Zeeland Journal of Obstetrics and Gynaecology. 2001;42(2):202-206

28. Dunaif A, Sorbara L, Delsan R, et al. Ethnicity and polycystic ovary syndrome are associated with independent and additive decreases in insulin action in CaribbeanHispanic women. 1993;42(10):1462-1468. doi:10.2337/ diab.42.10.1462.

29. Glintborg D, Mumm H, Hougaard D, et al. Ethnic differences in Rotterdam criteria and metabolic risk factors in a multi-ethnic group of women with PCOS studied in Denmark. Clin Endocrinol. 2010;73(6):732-738. doi: 10.1111/j.1365-2265.2010.03873.x.

30. Chen X, Yan D, Li L, et al. Abnormal glucose tolerance in Chinese women with polycystic ovary syndrome.. Hum Reprod. 2006;21(8):2027-2032. doi:10.1093/humrep/ del142.

31. Baba T, Endo T, Sata F, et al. Polycystic ovary syndrome is associated with genetic polymorphism in the insulin signalling gene IRS-1 but not ENPP1 in a Japanese population. Life Sci. 2007;81(10):850-854. doi:10.1016/j. lfs.2007.07.023.

32. Wijeyaratne $\mathrm{CN}$, Niranthara KK, Balen AH, et al. Plasma homocysteine in polycystic ovary syndrome: does it correlate with insulin resistance and ethnicity? Clin Endocrinol. 2010;73(6):732-738. doi:10.1111/j.13652265.2004.02019.x

33. Glueck CJ, Papanna R, Wang $\mathrm{P}$, et al. Incidence and treatment of metabolic syndrome in newly referred women with confined PCOS. Metabolism. 2003;52(7):908-915. doi:10.1016/S0026-0495(03)00104-5.

34. Wild RA. Dyslipidaemia in PCOS. Steroids. 2012;77(4):295299. doi: 10.1016/j.steroids.2011.12.002.

35. Wild RA, Carmina E, Kandarakis ED, et al. Assessment of cardiovascular risk and prevention of cardiovascular disease in women with PCOS. A Consensus disease statement by the Androgen Exess and PCOS (AE-PCOS) Society. J Clin Endocrinol Metab. 2010;95(5):2038-2049. doi:10.1210/ jc. 2009-2724.

36. Azziz R, Carmina E, Chen Z, et al. Polycystic ovary syndrome. Nat Rev Dis Primers. 2016;2:16057. doi: 10.1038/nrdp.2016.57.

37. Elsenbrunch S, Hahns S, Kowalsky D, et al. Quality of life, psychosocial well-being, and sexual satisfaction in women with polycystic ovary syndrome. J Endocrinol Metab. 2003;88(12):5801-5807. doi:10.1210/jc.2003-030562

38. Park JC, Kim SM, Rhee JH, Kim JI. Emotional stress and associated factors in women with Polycystic ovary syndrome. Ginekol Pol. 2016;87(6):405-10. doi:10.1016/j. fertnstert.2014.08.889

39. Setji TL, Brown AJ. Polycystic ovary syndrome: diagnostic and treatment. Am J Med. 2007;120:128-132. doi:10.1016/j. amjmed.2006.06.29

40. Legro RS, Barnhart HX, Schlaff WD, et al. Clomiphene, metformin, or both for infertility in the polycystic ovary syndrome. N Eng J Med. 2007;356:551-556. doi:10.1056/ NEJMoa063971

41. Lord MJ, Flight IHK, Norman RJ. Metformin in polycystic ovarian syndrome: systematic review and meta-analysis. BMJ. 2003;327:310-315. doi:10.1136/bmj.327.7421.951.

42. Lord JM, Flight IH, Norman RJ. Insulin-sensitizing drugs (metformin, troglitazone, rosiglitazone, pioglitazone, D-chiro-inositol) for polycystic ovarian syndrome. Cochrane Database Syst Rev. 2003;(3):CD003053.

43. Gjonnaess $H$. Ovarian electrocautery in the treatment of women with polycystic ovary syndrome (PCOS): Factors affecting the results. Acta Obstet Gynecol Scand. 1994;3(5):407-412. doi:10.3109/00016349409006253.

44. Tarlatzis BC, Fauser BC, Legro RS, et al. Consensus on infertility treatment related to polycystic ovary syndrome. Hum Reprod. 2008;23(3):462-477. doi: 10.1093/humrep/ dem426

45. Consensus on infertility treatment related to polycystic ovary syndrome. The Thessaloniki ESHRE/ASRMSponsored PCOS Consensus Workshop Group. March 2-3, 2007, Thessaloniki, Greece. Fertil Steril. 2008;89(3):505522. doi: 10.1093/humrep/dem426.

46. Moran LJ, Noakes M, Clifion PM, Tomlinson L, Galletly, Norman RJ. Dietary composition in restoring reproductive and metabolic physiology in overweight women with polycystic ovary syndrome. J Clin Endocrinol Metab. 2003; 88:812-819. doi: 10.1210/jc.2002-020815

47. Moran LJ, Brinkworth G, Noakes M, Norman RJ. Effects of lifestyle modification in polycystic ovarian syndrome. Reprod Biomed Online. 2006; 12:569-578. doi: 10.1016/ S1472-6483(10)61182-0.

Copyright (C) 2017 The Author (s); This is an open-access article distributed under the terms of the Creative Commons Attribution License (http://creativecommons.org/licenses/by/4.0), which permits unrestricted use, distribution, and reproduction in any medium, provided the original work is properly cited. 